\title{
Protein interaction networks as metric spaces: a novel perspective on distribution of hubs
}

\author{
Emad Fadhal ${ }^{1}$, Junaid Gamieldien ${ }^{1}$ and Eric C Mwambene ${ }^{2^{*}}$
}

\begin{abstract}
Background: In the post-genomic era, a central and overarching question in the analysis of protein-protein interaction networks continues to be whether biological characteristics and functions of proteins such as lethality, physiological malfunctions and malignancy are intimately linked to the topological role proteins play in the network as a mathematical structure. One of the key features that have implicitly been presumed is the existence of hubs, highly connected proteins considered to play a crucial role in biological networks. We explore the structure of protein interaction networks of a number of organisms as metric spaces and show that hubs are non randomly positioned and, from a distance point of view, centrally located.

Results: By analysing how the human functional protein interaction network, the human signalling network, Saccharomyces cerevisiae, Arabidopsis thaliana and Escherichia coli protein-protein interaction networks from various databases are distributed as metric spaces, we found that proteins interact radially through a central node, high degree proteins coagulate in the centre of the network, and those far away from the centre have low degree. We further found that the distribution of proteins from the centre is in some hierarchy of importance and has biological significance.

Conclusions: We conclude that structurally, protein interaction networks are mathematical entities that share properties between organisms but not necessarily with other networks that follow power-law. We therefore conclude that (i) if there are hubs defined by degree, they are not distributed randomly; (ii) zones closest to the centre of the network are enriched for critically important proteins and are also functionally very specialised for specific 'house keeping' functions; (iii) proteins closest to the network centre are functionally less dispensable and may present good targets for therapy development; and (iv) network biology requires its own network theory modelled on actual biological evidence and that simply adopting theories from the social sciences may be misleading.
\end{abstract}

Keywords: Protein interaction networks, Metric spaces, Core-periphery structure, Topological centrality, Hubs, Essential proteins, Power-law graphs

\section{Background}

In the post-genomic era, an overarching question in the analysis of protein-protein interaction (PPI) networks continues to be whether biological characteristics and functions of proteins such as lethality, physiological malfunctions and malignancy are intimately linked to the topological role proteins play in the network [1,2]. It has been established that protein interaction networks (PINs) have small-world and scale-free properties [3]. Much of the recent efforts in the analysis of protein-protein

\footnotetext{
*Correspondence: emwambene@uwc.ac.za

2 Department of Mathematics and Applied Mathematics, University of the Western Cape, P/Bag X17, Bellville, South Africa

Full list of author information is available at the end of the article
}

interaction networks has focused on finding functional dependencies between the so-called hubs and their topological roles in the network $[4,5]$. What has made the effort the more difficult is that various researchers have defined hubs from various points of view [6]. However, almost universally, it has been assumed that these hubs are proteins having a high degree (number of interactions) and that are randomly placed in the network and have important functional roles [4,7-10]. In a sense, it has been assumed that each hub or a specialised set of hubs somehow controls a sub-network that may constitute a pathway, functional module or a process [11-13]. Because of this, in defining many of the proposed metrics in PINs, the degree of nodes has prominently featured. It is appealing to give substantial import to these metrics because some of them 
show levels of statistical significance that may not easily be dismissed.

In that respect, for instance, it has been shown that in PINs, proteins of high degree are three times more likely to be lethal than the other proteins [5]. Due to this observation, network theory metrics such as degree centrality, closeness centrality [14], betweenness centrality [15], and cluster coefficients [16], to name a few, have been proposed as ways to identify functionally important proteins in PINs. Whilst these metrics have their relevance, they do not enable the evaluation and analysis of networks as topological entities. Many previous studies on the analysis of complex network used closeness centrality as a way to find the core central proteins and applied it in several areas such as to extract the metabolic core of a network [17], to visualise large scale complex networks in two dimensions [18], to identify drug targets [19,20], to identify essential genes in Escherichia coli [21] and to determination of dynamics of the cell-cycle networks [22]. Moreover, they used centrality measures which ultimately give weighting to nodes and do not attempt to identify the exact positions of nodes within the network [23]. Previously considered metrics have, however, implicitly assumed randomness of the distribution of hubs [3,24,25]. Because of the commonality in distribution of networks that follow power-law, some have gone as far as considering PINs in the same manner as social networks [26]. In point of fact, networks of protein interactions have been shown in separate organisms to form, in varying degree of statistical significance, scale-free networks in which the distribution of degrees of nodes is power-law $[27,28]$. It has thus been assumed that this property captures the essence of the pertinent features of the networks. In this article, it will become evident that paralleling the systems theory that has been developed in social networks to biological networks may not reveal a clearer picture.

The point of departure from these previous studies is that we model PPI graphs as metric spaces, which are welldefined topological spaces with a long history and deep theory $[29,30]$. This precise strategy provides a powerful way to view PINs in their entirety from a spatial point of view, using distance as the key modeling measure. While we do not attempt to weight proteins, it enables us to pinpoint exactly where nodes are located in respect to each other, even in very large PINs with several hundred thousands of proteins and many interactions. By identifying the network centre(s) using a formal method that identifies the protein(s) that have the smallest maximal distance to other proteins in the network, and then categorizing all proteins into zones based on distance from the centre, we are able to find exactly where any protein is located relative to the centre and its corresponding neighbours in the network at large. We show here that modeling and analysing PINs from several sources and organisms in this much more precise manner than can be achieved with centrality metrics reveals deep shared core-periphery topological pattern, and we also present strong evidence of its functional significance.

\section{Results and discussion}

\section{Multiple protein interaction networks from different} organisms all have single topological centres

While the Human Functional Protein Interaction Network (HPFIN), the Human Signalling Network (HSN) and multiple sources of Saccharomyces cerevisiae, Arabidopsis thaliana and Escherichia coli PINs all appear to be power-law distributed as expected (see Additional file 1: Figures S1a, S1b, S1c, S1d and S1e), we modelled each one of the PINs as metric spaces in order to identify each network's topological centre(s) and to classify remaining proteins into 'zones' based on graph theoretic distance from the central protein. Zone 1, for example, refers to proteins which are 1 step from the topological centre, zone 2 is 2 steps away, etc. By modelling the giant component of each PIN in this manner, we found that despite our method's inevitable ability to identify multiple topological centres if they exist, all PINs analysed had only a single central protein that have key biological functions. The centres of the HFPIN and the HSN, MAPK14 and MAPK1 respectively, belong to the same protein family and play similar key roles in signal transduction [31] and the centre of the Arabidopsis thaliana PIN is AT1G78300, which plays a key role in brassinosteroid mediated signaling [http://www.ncbi.nlm.nih.gov/pubmed/17681130]. Saccharomyces cerevisiae has SSC1, involved in stress response [http://www.molbiolcell.org/content/22/5/541. full] at the centre of its PIN and Escherichia coli has rpsB, a ribosomal protein $\mathrm{S} 2$ that is an essential component of the organisms translation machinery [http://www.ncbi. nlm.nih.gov/pubmed/23104805].

\section{PINs analysed as metric spaces form a dense core/sparse periphery structure}

The majority of proteins in PINs are located in zones $1-3$. For the HFPIN and the HSN respectively, $92 \%$ and 95\% proteins are in these zones. The same phenomenon is observed in Saccharomyces cerevisiae, Arabidopsis thaliana and Escherichia coli, albeit with varying proportionality (Table 1). When analysing zones as induced sub-networks, we found in all cases that zone 1 is the most highly connected with few quills, if any. Zone connectivity decreases with increasing distance from the centre, with zone 2 having some quills (nodes with degree 1 ) and zone 3 having many more quills (see the numbers of quills in Table 1). Beyond zone 3, due to very low connectivity, the induced subgraphs disintegrate into many components and in the zones on the fringes there are only quills. 
Table 1 Degree distribution of PINs with respect to the centre

\begin{tabular}{|c|c|c|c|c|c|c|c|c|c|c|c|c|c|c|}
\hline \multirow[t]{2}{*}{ Organism } & \multirow[t]{2}{*}{ Nodes } & \multirow[t]{2}{*}{ Edges } & \multirow[t]{2}{*}{ Diameter } & \multirow[t]{2}{*}{ Centre } & \multicolumn{10}{|c|}{ Zones around centre } \\
\hline & & & & & 1 & 2 & 3 & 4 & 5 & 6 & 7 & 8 & 9 & \\
\hline & & & & & 374 & 4610 & 3464 & 578 & 104 & 14 & 2 & 1 & 1 & Nodes \\
\hline & & & & & 86 & 32 & 52 & 2 & 2 & 1 & 1 & 2 & 1 & Average degree \\
\hline \multirow[t]{5}{*}{ Homo sapiens } & 9448 & 181706 & 13 & MAPK14 & 3 & 1 & 1 & 1 & 1 & 1 & 1 & 2 & 1 & Lowest degree \\
\hline & & & & & 531 & 430 & 393 & 14 & 6 & 2 & 2 & 2 & 1 & Highest degree \\
\hline & & & & & 0 & 173 & 653 & 307 & 56 & 12 & 1 & 0 & 1 & No of quills \\
\hline & & & & & 431 & 3527 & 1929 & 206 & 38 & 4 & & & & Nodes \\
\hline & & & & & 67 & 24 & 7 & 2 & 2 & 3 & & & & Average degree \\
\hline \multirow[t]{5}{*}{ HSN } & 6291 & 62737 & 11 & MAPK1 & 1 & 1 & 1 & 1 & 1 & 1 & & & & Lowest degree \\
\hline & & & & & 451 & 362 & 89 & 11 & 9 & 5 & & & & Highest degree \\
\hline & & & & & 4 & 404 & 757 & 133 & 20 & 2 & & & & No of quills \\
\hline & & & & & 175 & 1639 & 2517 & 565 & 69 & 9 & & & & Nodes \\
\hline & & & & & 34 & 14 & 4 & 1 & 1 & 1 & & & & $\overline{\text { Average degree }}$ \\
\hline \multirow[t]{5}{*}{ Saccharomyces cerevisiae } & 5033 & 22417 & 10 & SSC1 & 1 & 1 & 1 & 1 & 1 & 1 & & & & Lowest degree \\
\hline & & & & & 209 & 282 & 109 & 11 & 4 & 1 & & & & Highest degree \\
\hline & & & & & 1 & 92 & 609 & 327 & 57 & 9 & & & & No of quills \\
\hline & & & & & 134 & 216 & 799 & 825 & 443 & 130 & 20 & 5 & 4 & Nodes \\
\hline & & & & & 3 & 11 & 6 & 3 & 2 & 1 & 1 & 3 & 2 & Average degree \\
\hline \multirow[t]{5}{*}{ Arabidopsis thaliana } & 2953 & 6783 & 16 & AT1G78300 & 1 & 1 & 1 & 1 & 1 & 1 & 1 & 1 & 1 & Lowest degree \\
\hline & & & & & 62 & 117 & 79 & 43 & 26 & 12 & 4 & 6 & 3 & Highest degree \\
\hline & & & & & 81 & 60 & 180 & 355 & 234 & 80 & 10 & 1 & 1 & No of quills \\
\hline & & & & & 151 & 1089 & 976 & 260 & 38 & 6 & & & & Nodes \\
\hline & & & & & 37 & 12 & 3 & 1 & 1 & 1 & & & & Average degree \\
\hline \multirow[t]{3}{*}{ Escherichia coli } & 2949 & 12689 & 12 & rpsB & 1 & 1 & 1 & 1 & 1 & 1 & & & & Lowest degree \\
\hline & & & & & 178 & 127 & 56 & 6 & 3 & 2 & & & & Highest degree \\
\hline & & & & & 1 & 213 & 349 & 166 & 30 & 5 & & & & No of quills \\
\hline
\end{tabular}


An interesting pattern emerged when we assessed the distribution of the top $5 \%$ most highly connected proteins of each network across its zones. Overall, we see that these proteins coagulate in zones closer to the centre of the networks (Tables 2, 3, 4, 5, 6 and 7). When removing a potentially erroneous dense complete subgraph in zone 3 of the HFPIN consisting of 330 zinc finger proteins, the majority of the most highly connected proteins are located in zones 1 and 2. The same is observed for both the HSN and E. coli. While Saccharomyces and Arabidopsis PINs have a similar general pattern, they also have some of these proteins in zone 3, with the latter having an additional 9\% in its zones 4 and 5 .

It is therefore clear that across the phyla and regardless of network size, PINs form a structure that has a densely connected kernel and a less dense periphery, which terminates in 'quills' or 'spikes' (Figure 1). Furthermore, topologically central zones are highly connected and have only few proteins of low degree, which suggests a non-random distribution of hub proteins. It has previously been shown that evolutionarily older proteins have higher degree [32] and that loss and gain of protein-protein interaction sites are driven by evolutionary mechanisms [33] and therefore constrained by positive (Darwinian) and negative (purifying) selection. We therefore deem it reasonable to conclude that the core-periphery topologies uncovered by our method are themselves evolved and have functional significance and importance.

There is no phenomenal change (see Additional file 1: Figures S2, S3a, S3b, S3c and S3d) when the analysis is done on different PINs from different database sources. All the results from the analysis of PINs when treated as metric spaces confirm that PINs have a densely connected kernel and becomes less dense towards the periphery, terminating in several 'spikes' or 'quills'.

\section{Protein network topologies are not consistent with random graphs}

The striking similarity across the kingdoms suggests that these network topologies have functional significance and are evolved rather than random, especially since the number of proteins and interactions differ widely. In order to test this hypothesis, we performed a comparison of the

Table 2 Distribution of top $5 \%$ highest degree in human signalling network

\begin{tabular}{ccc}
\hline Zone & Number of nodes has degree $\mathbf{\geq} \mathbf{9 5} \mathbf{( 5 \% )}$ & Percentage \\
\hline Zone 1 & 106 & $34.6 \%$ \\
\hline Zone 2 & 197 & $64.4 \%$ \\
\hline Zone 3 & 0 & $0 \%$ \\
\hline Total & 306 & $100 \%$ \\
\hline
\end{tabular}

Table 3 Distribution of top 5\% highest degree in HFPIN without complete graph

\begin{tabular}{ccc}
\hline Zone & Number of nodes has degree $\mathbf{Z} \mathbf{2 0 0} \mathbf{( 5 \% )}$ & Percentage \\
\hline Zone 1 & 39 & $54.9 \%$ \\
\hline Zone 2 & 32 & $45.1 \%$ \\
\hline Zone 3 & 0 & $0 \%$ \\
\hline Total & 71 & $100 \%$ \\
\hline
\end{tabular}

biological networks to a large number of computationallygenerated uniform random power law graphs [34] with similar properties in terms of numbers of nodes and edges (interactions). In general, while all PINs have a single centre and large diameter, their random power-law equivalents often have multiple centres and significantly smaller diameters. PPI datasets also represented many components with a single giant component, while the random graphs consistently had at most 2 components. The number and distribution of quills, nodes of degree 1 , is also remarkably different, with PINs having a high number of nodes with low degree in comparison to uniform random power-law graphs (Table 8). This distorts archetypical power-law distribution of nodes. Furthermore, each PIN has a small number of nodes that have remarkably higher degrees than the highest degree nodes of the uniform random power-law graphs (Figures 2 and 3). The significant incongruence of topological patterns between PINs and random graphs add further plausibility to our hypothesis that the biological networks arose through positive selection.

\section{Central zones of human PINs are functionally specialised}

In order to assess whether the observed topological patterns have potential functional significance, we performed pathway enrichment analysis and observed strong zonespecific functional enrichment in the first four zones of the HFPIN and the HSN. Moreover, those zones also appear to be functionally specialised with most proteins in a zone belonging to the top four enriched pathways, while the outer zones are much more functionally diversified (Table 9, Additional file 1: Table S1). Zone 1 is highly enriched for proteins involved in signal transduc-

Table 4 Distribution of top 5\% highest degree in HFPIN with complete graph

\begin{tabular}{ccc}
\hline Zone & Number of nodes has degree $\mathbf{Z} \mathbf{2 0 0} \mathbf{( 5 \% )}$ & Percentage \\
\hline Zone 1 & 39 & $8.5 \%$ \\
\hline Zone 2 & 32 & $7.6 \%$ \\
\hline Zone 3 & 380 & $83.9 \%$ \\
\hline Total & 458 & $100 \%$ \\
\hline
\end{tabular}


Table 5 Distribution of top $\mathbf{5 \%}$ highest degree in Saccharomyces cerevisiae

\begin{tabular}{ccc}
\hline Zone & Number of nodes has degree $\geq \mathbf{3 5} \mathbf{( 5 \% )}$ & Percentage \\
\hline Zone 1 & 65 & $26.5 \%$ \\
\hline Zone 2 & 152 & $62 \%$ \\
\hline Zone 3 & 28 & $11.4 \%$ \\
\hline Total & 245 & $100 \%$ \\
\hline
\end{tabular}

tion, immune system, hemostasis and disease pathways and appears to constitute a core of highly important interactions required for organismal and cellular sensing and response to adverse environmental, biological and mechanical stresses. Zone 2 is also enriched for proteins involved in signal transduction and immune system pathways and is moderately enriched for gene expression and metabolic pathways, which are the main functional themes in zone 3 . Zone 4 has significantly less enrichment than zones closer to the centre, with metabolism and membrane trafficking being the main functional themes for HFPIN and HSN, respectively. Based on pathway enrichment observed in each zone and the high degree of functional specialisation observed in zones closest to the network topological centre, it is likely that the structure of the HFPIN and the HSN ( and possibly those of other organisms) may have strong biological significance. We propose that proteins closest to the network centre play critical roles in organismal survival (Figure 4, Additional file 1: Figure S4).

\section{Topologically central proteins may play critical roles in adaptation and survival}

In addition to the evidence presented for the human PINs, GO enrichment analysis of central zones of the Saccharomyces cerevisiae PIN appears to support our hypothesis that centrally located proteins may be important for organismal fitness, since zone 1 is enriched for functions related to cell cycle, response to stress, reproduction and response to DNA damage and zone 2 for functions related to RNA processing, chromosome organization, ribosome biogenesis and the mitotic cell cycle (Additional file 1: Figure S5). This is further reinforced by our findings that

Table 6 Distribution of top 5\% highest degree in Escherichia coli

\begin{tabular}{ccc}
\hline Zone & Number of nodes has degree $\mathbf{Z} \mathbf{4 4} \mathbf{( 5 \% )}$ & Percentage \\
\hline Zone 1 & 46 & $37 \%$ \\
\hline Zone 2 & 77 & $62 \%$ \\
\hline Zone 3 & 1 & $0.8 \%$ \\
\hline Total & 124 & $100 \%$ \\
\hline
\end{tabular}

Table 7 Distribution of top 5\% highest degree in Arabidopsis thaliana

\begin{tabular}{ccc}
\hline Zone & Number of nodes has degree $\geq \mathbf{~ 1 6 ~ ( 5 \% ) ~}$ & Percentage \\
\hline Zone 1 & 2 & $1.5 \%$ \\
\hline Zone 2 & 46 & $33.3 \%$ \\
\hline Zone 3 & 64 & $44.1 \%$ \\
\hline Zone 4 & 12 & $8.5 \%$ \\
\hline Zone 5 & 5 & $3.8 \%$ \\
\hline Total & 129 & $100 \%$ \\
\hline
\end{tabular}

topologically central positions of PINs are highly connected and that hub proteins are located in central zones. Topologically, this is in line with the core and periphery structures described for PPI networks [35]. However, we further propose that the 'switching' of specialised functions between zones and the high degree of enrichment for signal transduction proteins in zones 1 and 2 suggests that the human PIN has evolved to optimise the sensing of stimuli at its central zones and to initiate a signal outward to peripheral zones, where transcriptional and subsequent metabolic responses are effected.

\section{Conclusion}

When PINs are formally modelled as metric spaces, it becomes clear that hub proteins are not distributed randomly and that the prevailing view that proteins interact randomly with hubs marshalling low degree proteins in processes and pathways needs a serious reconsideration. We show clearly that PIN structures across the phyla have densely connected kernels and become less dense towards the periphery, terminating in several 'spikes' or 'quills'. We argue that structurally PINs, and possibly other biological networks, are mathematical entities that share properties between organisms but not necessarily with other networks that follow power-law, such as social networks. As such, while applying systems theory developed in social networks to biological networks may have been convenient in and may have shed some light on interactomes, it is not sufficient to identify functional patterns in protein interaction networks, which we have shown to have a much deeper topology when considered as metric spaces. As our over-representation analysis has shown that zones of the human and Saccharomyces cerevisiae PINs have functional significance, we argue that interactomics needs its own network theory modelled on actual PPI data rather simply adopting theories from the social sciences.

We thus conclude that our strategy of formally and precisely evaluating PINs as metric spaces, with a focus on zones relative to the centre, may shed light on the key differences between expressed PPI networks in normal and diseased tissues. We propose that centrally located 


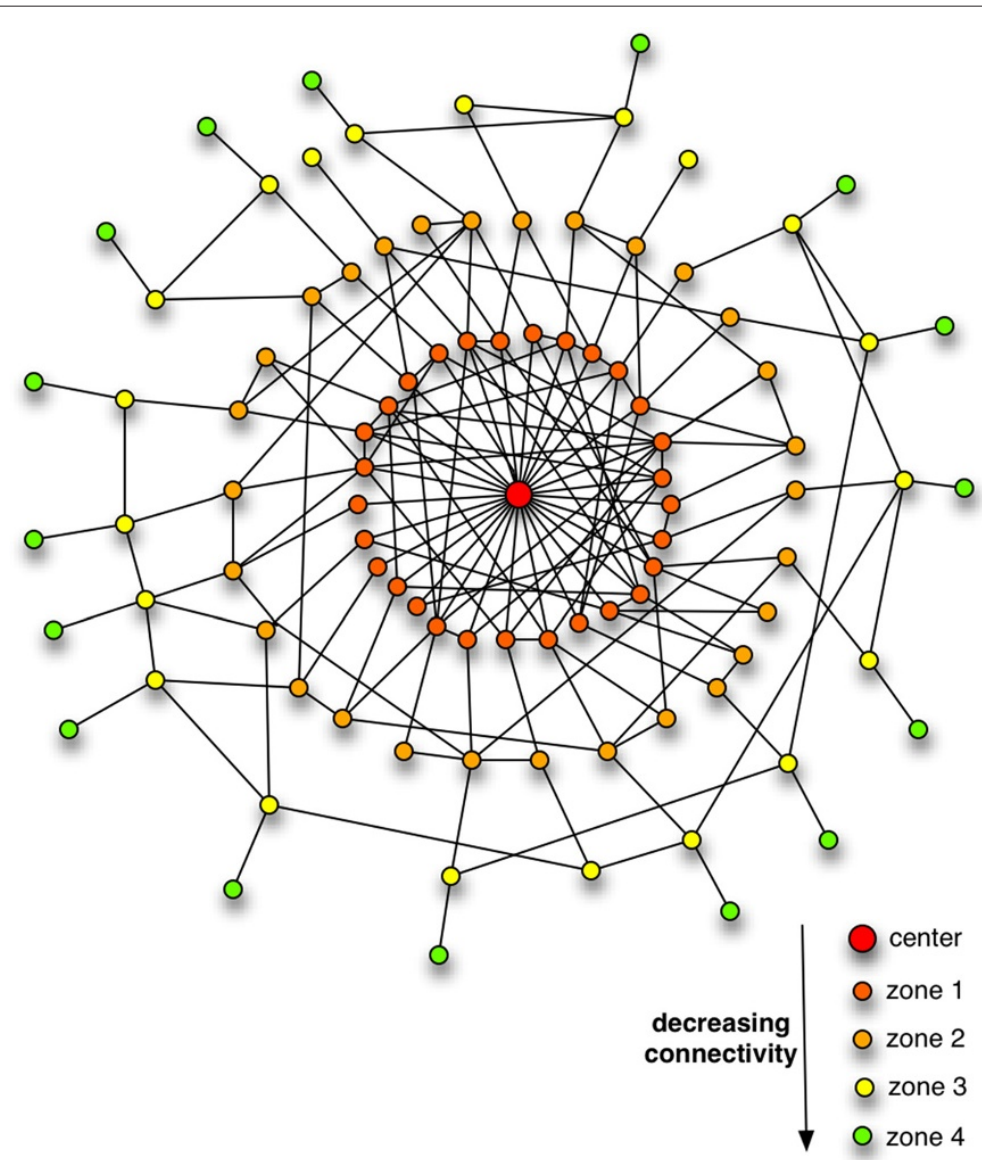

Figure 1 Model representation of a PINs with respect to distance from the centre. A graph layout representation of the PINs, which demonstrates a densely connected centre and 'quills' at the periphery.

proteins, particularly those involved in sensing functions, may present good therapeutic targets and should be formally evaluated in future studies based on our metric space approach. Our ongoing investigations into the potential applications of the approach detailed in this paper indicates that central zones of several human PPI networks are very strongly enriched for essential proteins and known drug targets, with central zones again displaying high enrichment (data not shown), reinforcing our hypothesis of utility in drug target discovery.

\section{Methods}

\section{Notation and definitions in graph theory}

The PPI networks we consider are modelled by graphs. A graph $G=(V, E)$ is a set $V$ together with an adjacency relation $E$ that is not reflexive and at the same time symmetric. The elements of $V$ are called nodes (one is a node) and those of $E$ are called edges. Thus, in PPI networks, proteins are represented by nodes and a pair of proteins forms an edge if they interact; we therefore interchangeably use proteins and nodes in this discussion. In the organisms we consider, to avoid reflexivity, we ignore considerations of proteins interacting with themselves. In any case, in matters of distances, reflexivity plays no role. The order of a graph is the number of its nodes and the size is the number of interactions. A graph is complete if every node is related to the other. If a node $x$ is related to a node $y$, we say that $y$ is adjacent to $x$ and write $x y$. The set of nodes that are adjacent to a node $x$ is the neighbourhood of $x$. The degree of $x$ is the number of nodes in its neighbourhood. In the context of PPI networks, the degree of a protein is the number of proteins that interact with it. A subgraph of a graph $G$ is a graph whose node set is a subset of that of $G$, and whose adjacency relation is also a subset of that of $G$. A subgraph $H$ of a graph $G$ is said to be induced if, for any pair of nodes $x$ and $y$ of $H, x y$ is an edge of $H$ if and only if $x y$ is an edge of $G$; that is, $H$ inherits all the edges that are in $G$. If a subgraph $H$ has only a subset of edges were defined in $G$, then $H$ is not induced. Of particular importance in PINs are induced subgraphs that define a process or pathway. A path in a graph is a sequence $v_{0} v_{1} \cdots v_{k}$ of distinct nodes such that every two consecutive nodes 
Table 8 Human signalling network vs equivalent random human signalling network

Network

Nodes

Edges

Giant

HSN

6291

62737

67

Random equiv. HSN 1

6477

64319

2

Random equiv. HSN 2

6270

62360

6267

62412

6277

62378

Random equiv. HSN 4

2

Random equiv. HSN 5

6282

62378

Diameter

11

\begin{tabular}{|c|c|c|c|c|c|c|}
\hline \multicolumn{7}{|c|}{ Zones around centre } \\
\hline 1 & 2 & 3 & 4 & 5 & 6 & \\
\hline 431 & 3527 & 1929 & 206 & 38 & 4 & Nodes \\
\hline 67 & 24 & 7 & 2 & 2 & 3 & Average degree \\
\hline 1 & 1 & 1 & 1 & 1 & 1 & Lowest degree \\
\hline 451 & 362 & 89 & 11 & 9 & 5 & Highest degree \\
\hline 4 & 404 & 757 & 133 & 20 & 2 & No of quills \\
\hline 155 & 3031 & 3149 & 134 & 5 & & Nodes \\
\hline 54 & 27 & 11 & 3 & 1 & & Average degree \\
\hline 7 & 1 & 1 & 1 & 1 & & Lowest degree \\
\hline
\end{tabular}

8

\begin{tabular}{cccccc}
\hline 150 & 141 & 61 & 10 & 1 & Highest degree \\
\hline 0 & 3 & 32 & 36 & 5 & No of quills \\
151 & 3037 & 2973 & 107 & 1 & Nodes \\
\hline
\end{tabular}

8

\begin{tabular}{cccccc}
151 & 3037 & 2973 & 107 & 1 & Nodes \\
\hline 56 & 27 & 11 & 2 & 1 & Average degree \\
\hline 5 & 1 & 1 & 1 & 1 & Lowest degree \\
\hline 159 & 136 & 50 & 6 & 1 & Highest degree \\
\hline 0 & 1 & 42 & 32 & 1 & No of quills
\end{tabular}

\begin{tabular}{cccccc}
\hline 0 & 1 & 42 & 32 & 1 & No of quills \\
\hline 171 & 3187 & 2812 & 96 & & Nodes \\
\hline 52 & 27 & 11 & 2 & & Average degree
\end{tabular}

7

\begin{tabular}{ccccc}
52 & 27 & 11 & 2 & Average degree \\
\hline 6 & 1 & 1 & 1 & Lowest degree \\
\hline 171 & 153 & 58 & 7 & Highest degree \\
\hline 0 & 1 & 39 & 33 & No of quills \\
\hline
\end{tabular}

\begin{tabular}{ccccc}
158 & 3127 & 2883 & 106 & Nodes \\
\hline 56 & 27 & 11 & 2 & Average degree \\
\hline
\end{tabular}

\begin{tabular}{cccccl}
\hline 5 & 1 & 1 & 1 & 1 & Lowest degree \\
\hline 153 & 143 & 54 & 7 & & Highest degree
\end{tabular}

7

\begin{tabular}{ccccc}
\hline 153 & 143 & 54 & 7 & Highest degree \\
\hline 0 & 2 & 29 & 36 & No of aulls
\end{tabular}

\begin{tabular}{cccccc}
154 & 3093 & 2914 & 119 & 1 & Nodes \\
\hline 55 & 27 & 11 & 3 & 1 & Average degree \\
\hline 4 & 2 & 1 & 1 & 1 & Lowest degree \\
\hline 153 & 158 & 53 & 8 & 1 & Highest degree \\
\hline 0 & 0 & 25 & 34 & 1 & No of quills \\
\hline
\end{tabular}




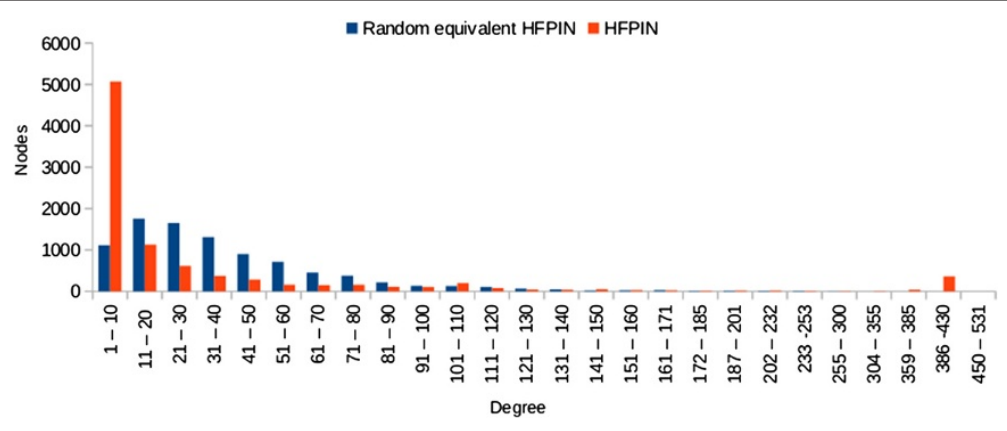

Figure 2 Degree distribution of HFPIN vs equivalent uniform random power-law graph.

constitute an edge in the graph; its length is $\mathrm{k}$. If for every pair of nodes in a graph there is a path joining them, we say that the graph is connected; otherwise it is disconnected. A component is a maximally connected subgraph of the graph and it is giant if contains a majority of the entire graph's nodes. The distance between a pair of nodes is the length of a shortest path joining them. A graph together with this distance defines a metric space. For a fixed node $v$, the eccentricity of $v$ is the length of the longest path joining itself to all the other nodes. The longest eccentricity of all nodes is the diameter of the graph and nodes with the shortest eccentricity are said to be at the centre of the graph. In our context, a quill is a subgraph which is on the fringes of the centre and eventually becomes a path, that is, a connected subgraph in which one of the nodes has degree 1.

\section{PPI data sources}

The human PIN we considered is the Human Functional Protein Interaction Network (HFPIN) [36], which has 9448 nodes and 181706 interactions. The human signalling network which has 6291 nodes and 62737 interactions was downloaded from www.bri.nrc.ca/wang/. PINs for other organisms were downloaded from various databases: the Database of Interacting Proteins (http:// bioinfo.esalq.usp.br) Version 02/28/2012 [37], bioGRID database (http://thebiogrid.org/download.php), CCSB interactome database (http://interactome.dfci.harvard. $\mathrm{edu} /$ ), antAnc database (ftp://ftp.ebi.ac.uk/pub/databases/ intact/current) Version 2.0, and MINT database (http:// mint.bio.uniroma2.it/mint/download.do). The Saccharomyces cerevisiae network consisted of 5033 nodes and 22417 interactions, the Arabidopsis thaliana network, 2953 nodes and 6783 interactions and the Escherichia coli PPI network consisted of 2949 nodes and 12689 interactions. In order to compare the biological graphs with random graphs, we generated uniform random powerlaw graphs that are similar in terms of number of nodes and interactions using the Python Webgraph Generator (http://pywebgraph.sourceforge.net/), which implements the RMAT algorithm [38].

\section{Evaluation of PPI networks as metric spaces}

We considered the Human Functional Protein Interaction Network, the human signalling network, the Saccharomyces cerevisiae, Arabidopsis thaliana, Escherichia coli, Caenorhabditis elegans and Helicobacter pylori PPI networks as metric spaces by defining the usual graph theoretic distance between nodes of a graph. Using a python wrapper around the $\mathrm{C}++$ BOOST graph library (http://

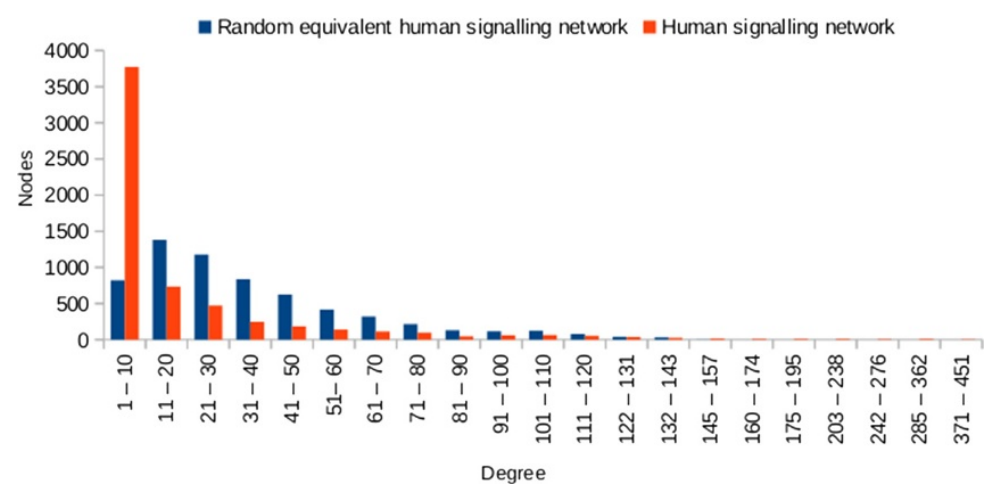

Figure 3 Degree distribution of human signalling network vs equivalent uniform random power-law graph. 
Table 9 Summary of functional specialization in the central zones of HFPIN

\begin{tabular}{|c|c|c|c|c|}
\hline \multicolumn{5}{|c|}{ Percentage of proteins } \\
\hline Enriched pathway & Zone 1 & Zone 2 & Zone 3 & Zone 4 \\
\hline Signal transduction & $38 \%$ & $24.8 \%$ & - & - \\
\hline Immune system & $29.8 \%$ & $10.6 \%$ & $4.5 \%$ & - \\
\hline Hemostasis & $17 \%$ & $5.7 \%$ & $2.7 \%$ & - \\
\hline Disease & $16.8 \%$ & $8 \%$ & $4.1 \%$ & - \\
\hline Gene expression & $8.2 \%$ & $8.8 \%$ & $9.8 \%$ & - \\
\hline Metabolism & $5.1 \%$ & $8.4 \%$ & $7.6 \%$ & $10.4 \%$ \\
\hline Membrane trafficking & - & - & $1.2 \%$ & $2.8 \%$ \\
\hline Neuronal system & $4.8 \%$ & $2.5 \%$ & $2.3 \%$ & $3.3 \%$ \\
\hline Transmembrane transport of small molecules & - & $1.9 \%$ & $2.4 \%$ & $3.6 \%$ \\
\hline
\end{tabular}

www.boost.org/), we used the Dijkstra algorithm to compute the shortest distances between all pairs of nodes and then identify the node or all nodes whose greatest distance to other nodes is/are smallest. This is the network center(s).

From here, nodes were classified according to their distances from the centre and divided into zones based on distance from the topological centre(s). From each distance class, we calculated their degree distributions and also considered their connectivity of the graphs induced for each zone.

\section{Pathway and function enrichment analysis}

In order to determine whether zones of the HFPIN, human signalling network and Saccharomyces cerevisiae
PIN we considered have biological significance, we divided proteins into subsets based on their distance from the true topological centre. Protein sets representing each zone were then subjected to a pathway overrepresentation analysis in order to determine whether the zones were specialised for specific functions. The Comparative Toxigenomics Database's Gene Set Enricher web service (http://ctdbase.org/tools/enricher.go) and Gene Ontology enrichment (http://www.geneontology.org/GO. tools) was used to perform the enrichment analysis and a corrected P-value of 0.01 was chosen as a statistical significance cutoff. Lastly, when such enrichment was observed, we calculated the proportion of proteins involved in each enriched pathway as a way to assess whether any zones display functional specialization.

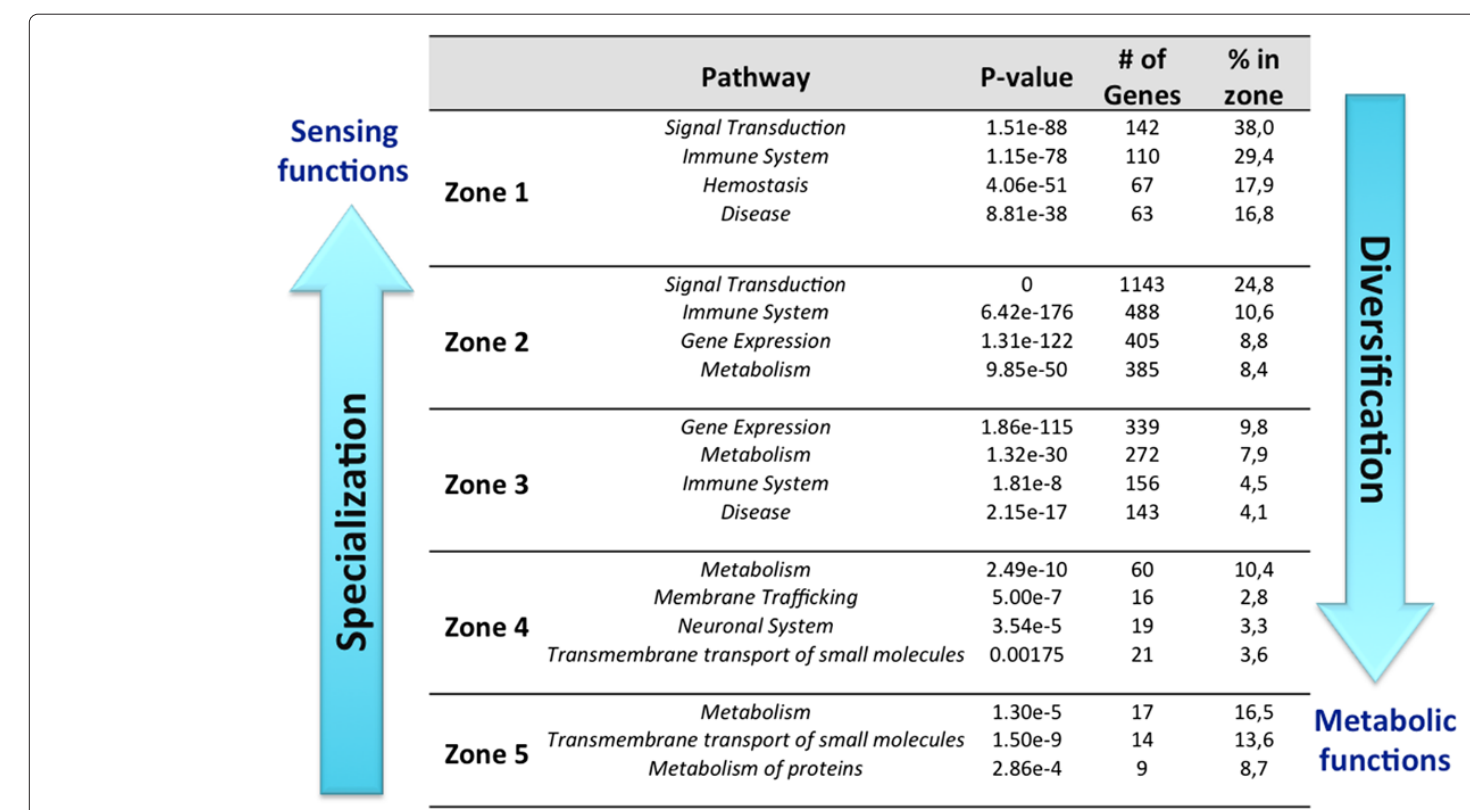

Figure 4 Summary of functional specialization in the central zones of HFPIN. 


\section{Additional file}

Additional file 1: Degree distributions of PINs. In all the PINs, the standard deviation of degree distribution has a remarkably significant variation. However, the HFPIN has a spike as a result of a zinc finger (ZNF) protein family of 330 proteins which constitutes an induced complete graph, where each protein has a degree of 386 . Our main strategy was to calculate a number of metrics of networks from their topological centre moving outwards. The correlation coefficient calculated for mean degree and the distance from the centre of the networks is $-0.789,-0.814,-0.840$, $-0.804,-0.865$, and -0.876 respectively for HFPIN, human signalling network, Saccharomyces cerevisiae, Escherichia coli, Caenorhabditis elegans and Helicobacter pylori. The correlation is therefore strongly negative. In other words, there is a relationship between mean degree and zones. As we move into the centre the values for the mean degree increase. On the other hand, the average degree of nodes in zones in the periphery decrease as one moves away from the centre. Figure S1a Degree distribution of the HFPIN. Figure S1b Degree distribution of Saccharomyces cerevisiae. Figure S1c Degree distribution of Arabidopsis thaliana. Figure S1d Degree distribution of Escherichia coli. Figure S1e Degree distribution of the HSN. Figure S2 Summary of degree distribution of PINs with respect to the centre from different sources. Figure S3a Degree distribution of HFPIN and human signalling network follows power-law distribution. Figure S3b Degree distribution of Saccharomyces cerevisiae from different source. Figure S3c Degree distribution of Arabidopsis thaliana from different source. Figure S3d Degree distribution of Escherichia coli from different source. Figure S4 Summary of functional specialization in the central zones of human signlling network. Figure S5 Summary of functional specialization in the central zones of Saccharomyces cerevisiae. Table S1 Summary of functional specialization in the central zones of human signalling network.

\section{Competing interests}

The authors declare that they have no competing interests.

\section{Authors' contributions}

EF developed the concept, implemented the algorithms and performed the analysis. ECM proposed the concept of analyzing the PPIs as metric spaces. JG oversaw the functional enrichment analysis and the biological interpretation thereof. ECM and JG designed and supervised the study. All authors have read and approved the final manuscript.

\section{Acknowledgements}

This work was supported in part by a grant from South Africa National Research Foundation (NRF)

\section{Author details}

${ }^{1}$ South African National Bioinformatics Institute, SA Medical Research Council Bioinformatics Unit, University of the Western Cape, Bellville 7535, South Africa. ${ }^{2}$ Department of Mathematics and Applied Mathematics, University of the Western Cape, P/Bag X17, Bellville, South Africa.

Received: 18 February 2013 Accepted: 7 January 2014

Published: 18 January 2014

\section{References}

1. $\operatorname{Kar} G$, Gursoy A, Keskin O: Human cancer protein-protein interaction network: a structural perspective. PLoS Comput Biol 2009, 5(12):e1000601.

2. Koyutürk M, Szpankowski W, Grama A: Assessing significance of connectivity and conservation in protein interaction networks. $J$ Comput Biol 2007, 14(6):747-764.

3. Barabasi AL, Oltvai ZN: Network biology: understanding the cell's functional organization. Nat Rev Genet 2004, 5(2):101-113. [http://www.ncbi.nlm.nih.gov/pubmed/14735121]

4. He X, Zhang J: Why do hubs tend to be essential in protein networks? PLoS Genet 2006, 2(6):e88.

5. Jeong $\mathrm{H}$, Mason S, Barabasi A, Oltvai Z: Lethality and centrality in protein networks. Arxiv preprint cond-mat/0105306 2001.
6. Vallabhajosyula R, Chakravarti D, Lutfeali S, Ray A, Raval A: Identifying hubs in protein interaction networks. PLoS One 2009, 4(4):e5344.

7. Batada N, Hurst L, Tyers M: Evolutionary and physiological importance of hub proteins. PLoS Comput Biol 2006, 2(7):e88.

8. Hahn MW, Kern AD: Comparative genomics of centrality and essentiality in three eukaryotic protein-interaction networks. Mol Biol Evol 2005, 22(4):803-806. [http://www.ncbi.nlm.nih.gov/ pubmed/15616139]

9. Yu H, Greenbaum D, Xin Lu H, Zhu X, Gerstein M: Genomic analysis of essentiality within protein networks. Trends Genet 2004, 20(6):227-231. [http://www.ncbi.nlm.nih.gov/pubmed/15145574]

10. Yu H, Kim PM, Sprecher E, Trifonov V, Gerstein M: The importance of bottlenecks in protein networks: correlation with gene essentiality and expression dynamics. PLoS Comput Bio/ 2007, 3(4):8. [http://www. ncbi.nlm.nih.gov/pubmed/17447836]

11. Zaki N, Berengueres J, Efimov D: Detection of protein complexes using a protein ranking algorithm. Proteins 2012, 80(10):2459-2468. [http://www.ncbi.nlm.nih.gov/pubmed/22685080]

12. Zaki N, Efimov D, Berengueres J: Protein complex detection using interaction reliability assessment and weighted clustering coefficient. BMC Bioinformatics 2013, 14:163. [http://www. pubmedcentral.nih.gov/articlerender.fcgi?artid=3680028\&tool= pmcentrez\&rendertype $=$ abstract]

13. Mete $M$, Tang F, Xu X, Yuruk N: A structural approach for finding functional modules from large biological networks. $B M C$ Bioinformatics 2008, 9(Suppl 9):S19. [http://www.ncbi.nlm.nih.gov/ pubmed/18793464]

14. Everett M, Borgatti S: The centrality of groups and classes. J Math Sociol 1999, 23(3):181-201.

15. Newman MEJ: A measure of betweenness centrality based on random walks. 2003. [http://arxiv.org/abs/cond-mat/0309045] [Comment: 15 pages, 7 figures, 2 tables]

16. Friedel CC, Zimmer R: Inferring topology from clustering coefficients in protein-protein interaction networks. BMC Bioinformatics 2006 7:519. [http://www.ncbi.nlm.nih.gov/pubmed/17137490]

17. Silva Md, Ma HMH, Zeng APZAP: Centrality, network capacity, and modularity as parameters to analyze the core-periphery structure in metabolic networks. Proc IEEE 2008, 96(8):1411-1420.

18. Ignacio AJ, DallAsta L, Barrat A, Vespignani A: k-core decomposition : a tool for the visualization of large scale networks. World Wide Web Internet And Web Info Syst 2005. abs/cs/050. [http://arxiv.org/abs/cs/ 0504107]

19. Vogelstein B, Lane D, Levine AJ: Surfing the $\mathbf{p} 53$ network. Nature 2000 408(6810):307-310

20. Jeong $\mathrm{H}$, Barab AL, Oltvai ZN: Prediction of protein essentiality based on genomic data. Complexus 2003, 1:19-28.

21. Lin CC, Juan HF, Hsiang JT, Hwang YC, Mori H, Huang HC: Essential core of protein-protein interaction network in Escherichia coli. $J$ Proteome Res 2009, 8(4):1925-1931.

22. Katzir Y, Elhanati Y, Averbukh I, Braun E: Dynamics of the cell-cycle network under genome-rewiring perturbations. Phys Biol 2013, 10(6):066001. [http://iopscience.iop.org/1478-3975/10/6/066001/article/]

23. Zhuge $H$, Zhang J: Topological Centrality and its e-Science Applications. J Am Soc Inf Sci Technol 2010, 61(9):1824-1841.

24. Mirzarezaee M, Araabi BN, Sadeghi M: Features analysis for identification of date and party hubs in protein interaction network of Saccharomyces Cerevisiae. BMC Syst Biol 2010, 4:172.

25. Patil $\mathrm{A}$, Kinoshita $\mathrm{K}$, Nakamura $\mathrm{H}$ : Hub promiscuity in protein-protein interaction networks. Int J Mol Sci 2010, 11(4):1930-1943. [http://www pubmedcentral.nih.gov/articlerender.fcgi?artid=2871146\&tool= pmcentrez\&rendertype $=$ abstract]

26. Bales ME, Johnson SB: Graph theoretic modeling of large-scale semantic networks. J Biomed Inform 2006, 39(4):451-464. [http://www. ncbi.nlm.nih.gov/pubmed/16442849]

27. Albert R, Barabasi AL: Statistical mechanics of complex networks. Rev Modern Phys 2002, 74:47. [http://www.springer.com/physics/theoretical,+ mathematical+\&+computational+physics/book/978-3-540-40372-2]

28. Watts DJ, Strogatz SH: Collective dynamics of 'small-world' networks. Nature 1998, 393(6684):440-442. [http://www.ncbi.nlm.nih.gov/ pubmed/9623998] 
29. Bryant V: Metric Spaces: Iteration and Application. Cambridge: Press Syndicate of the University of Cambridge; 1985.

30. Dugundji I: Topology. Boston: Allyn and Bacon; 1974.

31. Bandyopadhyay S, Chiang CY, Srivastava J, Gersten M, White S, Bell R, Kurschner C, Martin CH, Smoot M, Sahasrabudhe S, Barber DL, Chanda SK, Ideker T: A human MAP kinase interactome. Nat Methods 2010, 7(10):801-805. [http://www.ncbi.nlm.nih.gov/pubmed/20936779]

32. Eisenberg E, Levanon EY: Preferential attachment in the protein network evolution. Phys Rev Lett 2003, 91 (13):138701. [http://arxiv.org/ abs/cond-mat/0303490]

33. Gibson TA, Goldberg DS: Improving evolutionary models of protein interaction networks. Bioinformatics 2011, 27(3):376-382. [http://www. ncbi.nlm.nih.gov/pubmed/21067999]

34. Aiello W, Chung F, Lu L: A random graph model for power law graphs. Exp Math 2001, 10:53-66. [http://www.emis.ams.org/journals/EM/ restricted/10/10.1/aiello.ps]

35. Luo F, Li B, Wan XF, Scheuermann RH: Core and periphery structures in protein interaction networks. BMC Bioinformatics 2009, 10(Suppl 4):S8. [http://www.ncbi.nlm.nih.gov/pubmed/19426456]

36. $W u G$, Feng $X$, Stein $L:$ A human functional protein interaction network and its application to cancer data analysis. Genome Biol 2010, 11(5):R53.

37. Brandão MM, Dantas LL, Silva-Filho MC: AtPIN: Arabidopsis thaliana protein interaction network. BMC Bioinformatics 2009, 10:454. [http:// www.pubmedcentral.nih.gov/articlerender.fcgi?artid=2810305\&tool= pmcentrez\&rendertype $=$ abstract]

38. Chakrabarti D, Zhan Y, Faloutsos C: R-MAT: A recursive model for graph mining. Computer 2004, 6:442-446. [http://repository.cmu.edu/cgi/ viewcontent.cgi?article=1541\&context=compsci]

doi:10.1186/1752-0509-8-6

Cite this article as: Fadhal et al.: Protein interaction networks as metric spaces: a novel perspective on distribution of hubs. BMC Systems Biology 2014 8:6.

\section{Submit your next manuscript to BioMed Central and take full advantage of:}

- Convenient online submission

- Thorough peer review

- No space constraints or color figure charges

- Immediate publication on acceptance

- Inclusion in PubMed, CAS, Scopus and Google Scholar

- Research which is freely available for redistribution 